\title{
Human Factors in Automating Cyber Operations
}

\author{
Robert S. Gutzwiller \\ Arizona State University \\ rgutzwil@asu.edu
}

\author{
Dirk Van Bruggen \\ Laboratory for Advanced Cybersecurity Research
}

\begin{abstract}
Human-machine interaction issues must be addressed in designs and implementation of automation for cybersecurity. The community must not start from scratch to implement best practices; we review for the community and practitioners the relevant history and research done by human factors for human-automation interaction. We bring these theories and results to a handful of cybersecurity elements with two main goals: (1) educate the cyber discipline, and (2) provide guidelines toward building automated cybersecurity technology.
\end{abstract}

\section{Introduction}

The human role in cybersecurity operations is a major one. A lack of operators capable and qualified for the many job roles and demands of cybersecurity is a key problem to solve. Operators here can be viewed as the general cybersecurity workforce, which the NIST framework defines as "a workforce with work roles that have an impact on an organization's ability to protect its data, systems, and operations." For example, per [1] a Cyber Defense Analyst "uses data collected from a variety of cyber defense tools (e.g., IDS alerts, firewalls) to analyze events that occur within their environments for the purposes of mitigating threats." This role has been a major target for automation due to well-known problems of burnout and fatigue [2]. These are not the same as end-users, which have received plenty of research study [3], [4].

A solution to lack of staffing in many domains has been to implement automation into the work process with varying degrees of success. As cybersecurity aims to increase the efficacy of operations but struggles to meet its staffing targets, automation and autonomy represent two solutions being explored. As a science, human factors engineering has been influential in understanding what makes automation implementations successful or unsuccessful. These design principles have been demonstrated in other domains of high complexity, including aviation and process control environments but not yet applied to cyber.

We define two key terms here to clarify our discussion in this paper, automation, and autonomy. Automation: technology that actively selects data, transforms information, makes decisions, or controls processes (normally where humans used to do these tasks; [5]). Autonomy: automation that also 'thinks' and updates, trains itself, and is wholly responsible and authoritative for its actions completely absent a human. While automation is present across society especially in modern operations and technology environments, autonomy by this definition is not. We focus on automation as a more relevant near-term possibility. Autonomy is a goal identified in many strategic documents describing the future of operations. But it is also a long-term, and possibly overly optimistic futuristic goal. In contrast, automation in cybersecurity could improve operations in the near term. It may enable rapid reaction to detect and disrupt cyber-attacks before they do damage. However, the potential is mediated by the need to carefully consider human cognition and task performance in: (1) how the work may be affected by technology implementation, (2) how interactions with automation should be designed (3) deciding what tasks should be automated.

We saw an opportunity to educate current cybersecurity professionals, including those working on software, IT, and managing those activities (along with researchers), to help them avoid prior 'ironies' of automating activities. Secondly, the reviewed content leads to several best practices pulled from the field and discussed in the frame of cybersecurity which could be used by program developers, system architects and human systems integration experts to better design and develop new automation. These have not been traditionally applied to cybersecurity programs. Making this connection, in a rich and informative way, is the key contribution of this work, in addition to providing design recommendations that could improve the safety, security and performance of cyber systems. 


\section{Human automation interactions}

A common but fatal design flaw is the lack of attention paid by designers to the interaction of the system with the human who operates it. These elements are routinely ignored or left unstudied, because they are (incorrectly) viewed as unscientific, unhelpful, overly complex, out of scope, or simply difficult to understand. As with much of human behavior, members of the general population feel, having lived a human life, that they can understand the cognition of users, and make accurate predictions about how their system will be used [6]. Such a bias is made worse when these same people, taking the role of system creator, are intimately familiar with the actual design and operation of the system [7]. Biases are one topic area in cybersecurity, but investigations of them so far have not focused on automation [8], [9]. Particular attention has been given to events in which human interaction with automated systems resulted in catastrophe; in other words, a worthy system still had a negative outcome, and that outcome was linked to poor humanautomation interactions. The USS Vincennes incident in which a civilian airliner was shot down; Patriot missile battery friendly fire incidents in which allied aircraft were shot down [10]; recent US Navy ship collisions [11], [12], and countless unfortunate accidents in aviation and process control resulting in crashes and loss of life reveal an unfortunate message: in realistic human-automation interaction conditions, there has not been enough attention paid to how humans use, misuse or abuse these types of systems [13], [14].

While the examples from aviation and military events are not in the cyber realm, they have clear implications. Cybersecurity operations are also occurring in complex systems and organizations that often have numerous protections built in to avoid catastrophe. As in both military and aviation domains, cybersecurity has the potential for severe consequences. And finally, both domains rely on proper interaction with humans even with high levels of automation. The good news is that prior research has worked to improve these interactions and derived guiding principles for development. Human factors, psychology, cognitive engineering, human systems engineering, human systems integration, and its many other similar names brings with it this specialized understanding of the human-automation interaction problem. Yet these fields and their guidance have remained isolated from cybersecurity operations until very recently [15], despite work done in HCI on end user and developer security practices which is not applicable for the cybersecurity workforce [16], [17].

\subsection{Theoretical maturation and justification}

At its epistemological roots, automation has been defined as technology (computers, in the modern era) taking over what was once performed by a human [5], [18]. At fault for some of the incidents mentioned previously was an ideological perspective of separately identifying different agents" capabilities - the "man is better at, machine is better at" categories (see [19]). The abilities of people are different than machines; yet this is no substitute for a design philosophy. The belief that knowing who is best at a task should dictate who does it dates back to the misinterpretation of a 1951 technical report by Paul Fitts and colleagues ([20], p. 7-8). In this report, the authors attempted to represent differences between human and machine; but their goal was not to assign different work functions or roles (e.g., memory, repetition) to either human OR machine. As the ability of machines and computers began to grow, these conceptualizations were challenged, and changed in part because engineers had to confront actual users within increasingly complex systems. Nearly thirty years after the Fitts report (1951), the seminal report of Sheridan and Verplank in 1978 [21] further suggested many different, and potentially useful methods of shared control and augmentations between human and computer including extending, relieving, backing-up or replacing. Human and machine were no longer separate entities each doing some work in a siloed fashion. The report also developed the now widely known levels of automation, which are famously cited and remain central to discussions of human-machine interactions today. But ironically, they too, were only produced as a suggestion by the authors, an indication and provocation of the much larger variety of possible configurations; they were not to be treated as an algorithm, taxonomy, or limited set of options, or even as design guidelines [22]. Sheridan and Verplank helped lead engineers away from siloed agents in a system, but this was perhaps less intuitive in practice. And, because engineers gravitated to the levels-of-automation definition, new designs still resulted in problems. Many more modern methods and theory surrounding development of automation are now available; other 'levels' of automation scales are present in the literature [23].

A few modern updates [24], [25] move from levels to degrees of automation and tie the levels of automation to human performance across various functions (e.g., informing, executing, selecting). An abstracted, 4-stage information processing view of cognition was used [24], 
and the authors represent a given automated system as having different levels of automation, rather than one [21] and map this to human information processing (information acquisition, analysis, decision selection, and action implementation). Readers may recognize the information processing schema as similar to the "OODA" loop ([26]), and each has alignment with observation, orientation, decision making and action. The description allows a designer to recognize specific impacts on different 'stages' of human cognitive processes based on incorporating their designs (see Figure 1). Automated systems can also operate at high or low levels (i.e, doing more or less of the task) for each information processing stage. The framework can make a big impact both in design and interpretation of larger complex system operations. A recent meta-analysis showed for example, that automation failures for systems that partially or fully automate human decision-making and action-taking functions according to the Parasuraman et al. concept are particularly disruptive to human and overall system performance [27]; whereas some relatively high error rates from automated systems can be tolerable, and even useful, for information gathering, and analysis [28]. Thus, the information processing stages - as a characterization of what an automated system is doing for the human - helps to design an automated component; it helps the psychologists and human factors engineer understand how the automation effects that human process. The framework continues to be a landmark as a result, especially for its specific section on design methodology (discussed later).

\section{3. "Ironies of Automation"}

The instantiation of automation is not, itself, problematic. The conditions for ironies emerge around any tasks where a high degree of complexity, as well as unpredictability, combines with automation and co-location of that automation with a human operator. Bainbridge [29] discussed how automation created in industry often failed to meet its goals and instead made things worse. Though these were observed in industrial processes, they are more widespread, also occurring in domains such as transportation. Yet, they are not emphasized or widely known for cybersecurity. A few of the ironies revealed appear to be common sense; yet, in every case they are ideologically at odds with the old engineering philosophies of "automate everything the human cannot do well" or "automate everything that you can automate" which persist today in many realms. Former head of the National Transportation Safety Board (NTSB) Barry Strauch also noted that despite 35 years of awareness and study in aviation, major accidents involving automation continued to occur [14], a result no doubt because lessons had to be learned and it takes a very long time to apply them in that field. While human-automation interaction is broadly defined, where can the lessons of the past apply to applications of automation in cybersecurity? We call attention to the most prominent ironies of automation from the longer list by Bainbridge [29], and take the extra step to link them to cybersecurity.

\subsection{Irony 1: Changing the nature of work by deskilling, altering cognitive demand, and hiding operator deficits}

Adding automation fundamentally changes the task for the operators and the skills they need to succeed [30], [31]. It can lead to a "requirements shift" in individuals and staffing, training requirements, and can create (rather than remove) some taskwork that is poorly suited to humans, such as vigilant monitoring. In the cybersecurity space, many roles are already understaffed, and the definition of expertise itself unclear as is the process by which new experts are created. The narrower the realm of operations, the narrower the expertise required becomes. Introducing automation into a narrow operational context may reduce the skilled workforce that are in such high demand, or may at least reduce the ability of those experts to operate effectively after longer periods of automated performance. Little attention has been paid to this phenomena for cybersecurity, but pilots and aviation companies understand and recommend refreshing and retaining manual flight control skills. Automation can hide skill deficits [13]. Indeed some skill deficiencies emerge only under non-typical conditions, when the automation components cannot or do not perform, and instead the humans or other components of the system are under increased demand or authority. Unfortunately, automation can even create emergent conditions where the human must respond perfectly to avert disaster [29]; therein lies the irony: one begins automating to remove the role of the human and achieves it only to find that performance suffers potentially catastrophic collapse.

And on top of it all, due to the change in skill required, it is not that automation always reduces the staffing requirements. In some cases, it increases them or increases the need for more experienced, harder to find talent. Often, these effects go beyond individuals and narrow sets of operation. For cybersecurity, it is important to consider the broader, system wide effects. One clear example of the ironies of automation was in the implementation of the "optimal manning" program, which involved a massive amount of technology promising to reduce workforce required by the Navy 
through computers and automation. This program has since been rescinded and can be viewed to have caused exactly the above ironic consequences [32].

A growing area of investment in security organizations is that of security orchestration, automation and response (SOAR) solutions. Per Gartner, SOAR platforms enable organizations to collect monitoring data (e.g. alerts, logs, telemetry) in a central location where incident analysis and triage can be performed leveraging a combination of human and machine power to then drive standardized incident response activities [33]. Organizations hope that these systems will ease burdens on operators by centralizing all interactions into a single interface. However, these platforms introduce new skill requirements for the security organizations both to develop and integrate SOAR technologies into the existing infrastructure as well as to troubleshoot problems in these complex systems when they arise.

It is important that as such systems are rolled out, operators continue to train up in all of the underlying tools and technologies to ensure the maintenance of skills. Even more important is for new employees brought into the organization to undergo not only training on the underlying tools but also practice manual procedures to build up the muscle memory to ensure correct application of skills while under the pressure of non-standard situations. Organizations may understand some of these issues. When interviewed, only $8 \%$ of respondents believed that they would experience a reduction in staffing levels due to automation. However, almost 50\% expected that they would better utilize their current staff members [34]. In order to ensure automation systems live up to these expectations, organizations will need to act strategically to ensure tasks and operator skills are both developed with these risks in mind.

\subsection{Irony 2: Reduction and loss of situational awareness, and the challenges of monitoring automation.}

A system doing the work for a user, often still requires that operator to engage in monitoring of that work [35], [36]. No system can be built that is flawless; we often check the simplest of technologies, even our thermostats, despite their reliability. When designed, such a system is usually not viewed as work intensive, despite monitoring and vigilance behavior being highly mentally demanding [37]. It is assumed that with the automation doing the work, the human has additional time and mental resources they can allocate to other tasks. And therein lies the irony; a justification for bringing in automation is to relieve operators to do "more important" work. Yet often they are then required to monitor the system; to do this, operators now have to devote additional mental effort to tracking the automated work and if the system is highly effective or very quick in its actions, there will not - as a matter of design - be enough time for humans to verify [29].

Reduction and loss of situational awareness is a serious associated concern, and affects both training and practice as well as operational elements [38]-[40]. The use of automation can result in reduced awareness of the ongoing work [23], [41]. Simply put, automation can hide states of the system, along with critical information, which allows users to understand and make predictions about current and near-future states of the system [42], [43], and other processes rendering the operator less aware.

An example in the cybersecurity field can be seen with intrusion detection systems (IDS) and alert fatigue [2]. IDS were developed to automate the collection of data and detection of threats based on various types of network and endpoint data. However, due to the high volume of false positive alerts generated by IDS, tier 1 analysts spend most of their time monitoring these alerts, often without finding any true positives. A significant goal motivating adding automation is to reduce alert fatigue among analysts and allow them to devote more of their time to deeper investigations. Unfortunately, when organizations who have used automation are surveyed, only $36 \%$ are confident that automation would reduce alert fatigue [34].

In addition to difficulties in implementing IDS programs, it is well known that the verification and validation of these systems is a complex and potentially expensive process that often goes overlooked [44]. Therefore it could be argued that while the initial automation increased the situational awareness for analysts by continually checking for known threats, it also creates a false sense of security and a follow-on lack of awareness as to whether or not the system is, indeed, performing as intended.

While developing and maintaining situational awareness is a challenge across a multitude of domains, a remaining challenge in cybersecurity research has been to understand and quantify defender cognitive situational awareness (e.g., [45]-[47]). The effect on awareness of automated components or systems being introduced is rarely examined in the context of cybersecurity. But we can assume in general that poorly designed systems will reduce, not improve, cyber defenders' awareness unless shown otherwise. Further support for this can be found in the generally user-agnostic design and evaluation of other cybersecurity tools and displays, as shown in a review by Staheli and colleagues [48]. 
These ironies are the result of complex interactions between the humans and the system, where optimizing one aspect of operation can have unforeseen consequences; yet the persistence of the ironies suggests that at least some of the humanautomation interactions can be improved ahead of time by addressing them. These ironies can in effect be framed as "principles": they are tried and tested across multiple disciplines; they align with existing theory; they appear to occur for experienced and novice operators; they rely on behavioral evidence when people have skin in the game. Linking these principles to cyber automation and dissolving the automation-centric mindset in designers is beneficial.

\subsection{A concrete cybersecurity example of human automation interactions}

As discussed in Section 2.1, automation tasks can be described by using both levels and degrees of automation. Figure 1 shows a task may fall across the spectrum.

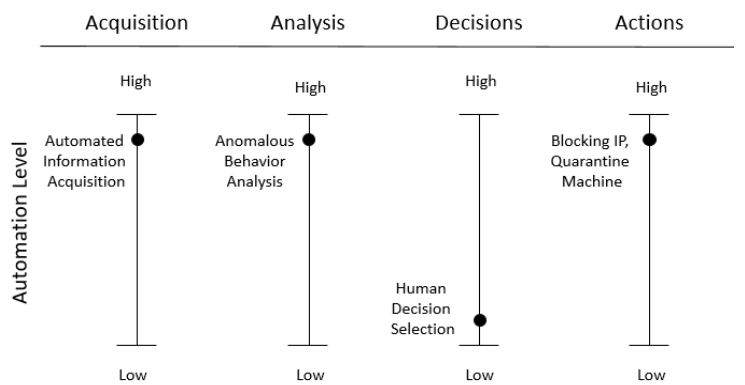

Figure 1: Comparison of levels and degrees of cybersecurity automation

An automated information acquisition program for cybersecurity may pull in vast quantities of data, which normally a person or team of people would have done by searching multiple sources to find and integrate. This system could be characterized as having a high level of automation, but a low degree of automation (it highly automates an earlier portion of the human information process). Once information is input, a highly automated analysis may begin which searches the data for certain properties (e.g., if the data were packet capture or connection logs, perhaps we are looking for anomalous behavior originating from an IP we do not usually see interactions with) - again, a high level of automation. However, for this analysis, now the degree of automation is increased because we move deeper into information processing.

Once an alert is triggered the human-machine system must reason on what to do (sometimes this is known ahead of time, but often, uncertainty and emergent conditions require some reflection on rules of engagement, strategy, etc.). Fully performed by the human, this requires no automation and is therefore low level and no degree. Finally, action is implemented (the action doesn't have to be extreme, like blocking the IP and quarantining the machines it touched - it could simply be to delay a decision until certain data emerges, or to go search for more information), creating an iterative cycle.

Action is partially automated at a low level (e.g., stages of actions linked together by various computer systems) but operating at a high degree of automation (action implementation). Higher degrees and higher levels may need more scrutiny than lower ones, as described before.

\section{Cybersecurity applications and practice}

The inability to make systems which have guaranteed behaviors results in systems which can inexplicably fail in unfortunate ways, particularly when under adversarial attack. Systems used to perform automated actions for cyber operations are often complex, making deep understanding very difficult even for experts. As a result, cyber systems tend to be completely trusted - until they are not. If the individual deficiency leading to the software or hardware failure can be mitigated, then we may recover our trust quickly; but multiple, correlated, or continuous failure, may lead to it being abandoned altogether.

While automation is present in almost all actions taken in a cyber environment, few to none of these operates without human intervention and guidance. Currently, even "manual" operations are mostly automated, but a human serves at the helm in supervisory role of a software application, which performs one or more actions in sequence. These automated aspects hide vast and complex sequences of automated steps by the software, operating system, and hardware on which it executes.

These feats of automation help defensive and offensive cyber operators to avoid manual overload. Operators not only make heavy use of pre-existing software automation mechanisms, but often develop a litany of custom scripts, tailored algorithms, and mission-specific procedures - each of which serves to improve their effectiveness many times over. Neither compensates well for tactical and strategic input by the operator (whether at setup, or ad-hoc during a mission), though they qualify as "automation".

For example, when an attacker considers how to scan a network, s/he has a range of options, from highly automated (but sometimes potentially "loud", detectable) scans, to very manual, low and slow "quiet" scans. The 
human is the arbiter calibrating the level of desired support of the machine, or the "level of automation". The information acquisition component could be highly automated by these scans - they may reveal most of the information. However, most by themselves may not do the 'analysis' portion (which is left to other programs, or operator assessment). Further, the operator is generally making the decision about the course of action. Actions themselves are usually taken by the operator. Of course, some types of defensive capabilities automate this entire process. And automation of data collection and interpretation to support reconnaissance for example, could be further explored and understood given humanautomation interaction theory and background.

Across cyber defense, some automation systems require the human to 'turn on' the automation capability and remain vigilant of alerts. For example, a SOAR platform allows for many different playbooks to be developed, and then can trigger them based on automated rule checking (e.g. all e-mails with attachments should be have the attachment fed into a malware sandbox before delivering the e-mail) or human driven commands. These are sort of expert systems approaches that may automate certain pieces or raise alerts up to humans to act. Allowing the human to make the choice of enabling automation can outperform other configurations (e.g., [49]). However, in cybersecurity applications we should be wary of conditions which may make the arrangement unstable. Firstly, surprises can disrupt operators and they may be unable (or simply forget) to turn on the automation as a result. While this makes little sense using the IDS system as an example, it may apply in more futuristic network posture situations, where automation is more prevalent. Imagine a case in which a unit or network senses attack and thus automatically engages a set of network-wide actions to protect critical assets from being disrupted further. Under normal conditions, these rules would be constricting other missions and goals of the organization, but under attack conditions they may save or preserve access to key resources. Knowing when to engage the rules may be handed to an automated controller, invoking the automation issues previously discussed. Second, people may choose not to rely on the machine, even when their own performance is suffering. Examples of ignoring or overriding systems at critical moments (as in the grounding of the Costa Concordia), suggest operators can easily become "overconfident" in their abilities, or underconfident in the system's abilities [50].

Software environments are particularly prone to automation being blamed for poor performance overall, even when performance without the automation - especially when such a system is under attack - is much worse. Leadership or management may also be vulnerable, since they are more removed from the potential feedback loops calibrating other front-line operators' behaviors and trust. In a recent survey of security operation center (SOC) professionals, $82 \%$ were confident in their ability to detect threats within their environment while less than $36 \%$ of professionals are actually tracking common performance metrics that could back up the confidence, such as mean time to detect (MTTD) or mean time to respond (MTTR) [51].

Careful consideration of where to place authority over automated actions is due, especially within an organization, as the authority may not understand what they are really doing. Mechanisms of ensuring trust (and blame) need to be sufficiently understood and traced to shore up feedback loops between performing agents (both automated and human), and better enable organizational learning.

The use of an adaptive automation system may offer some advantages to performance and awareness [52]. Adaptive automation systems may adjust configurations, performance parameters, or even aspects of the external environment based on user attention and cognitive load, potentially mitigating loss of awareness, offering reduced cognitive workload, and outperforming configurations lacking adaptive or shared control. They do not rely on the user to activate the system - they can turn themselves on, which solves some of the problems of adaptable automation. However they invoke other problems to be solved, such as determining when the automation triggers (by what mechanism), which has been the target of many research efforts [53], [54].

Leaving the implementation of automation - what does it do, when does it do it - up to the operator may be a bad idea. Yet taking it out of their hands is also problematic. A whole domain of study surrounding function allocation and authority can be consulted, but is hardly conclusive of how to arrange who does what, only on how to measure potential configuration [55]. There are ways of designing for the situation; but they are not simple processes (see Figure 1).

\section{Human factors in designing for automation in cybersecurity}

Recommendations for developing automation and autonomy in cybersecurity are still outstanding, and there are many reasons for this gap. First, while human investigations in cybersecurity appear prolific, as shown previously they are primarily concerned with end-user activity - whereas most predictions and capabilities being developed to automate security are at the professional operator level. Second, it is likely that specific recommendations would be inadequate in 
creating new systems. A common complaint against the study of the human systems integration and human factors realms is that the advice is often not broad enough; noting that these methods do not generate the specific guidelines needed to develop AI technology, and that they rely on the specifics for each unique system. This makes scaling very difficult for organizations like the military who want to integrate AI across mission areas, operators, devices, and authority structures. We have addressed that problem here by explicitly keeping the advice broad to allow for a variety of different cyber-related products to benefit from our best, global principles. This is a strength, not a weakness. Finally, the gap certainly indicates that more work is required, and this work should be a community effort - not only done by those with skill in IT and security, but also (and not separately) those with psychological expertise, usability, engineering and system integration as their expertise.

Parasuraman et al. (2000) developed a way to incorporate human information processing approach into some general guidelines for creating, identifying and evaluating different levels and degree of automation (Figure 1 below).These guidelines should be implemented after a task or job is understood from both the human, and more technical or computational perspectives. One would like some sense of function allocation - the 'who does what' - to be understood, explored, and defined throughout this process (Figure 2 ), and then evaluated by metrics [55].

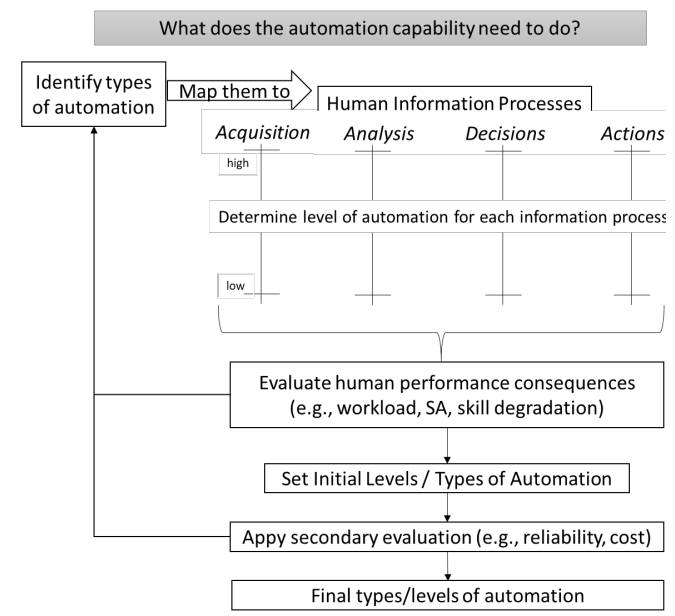

Figure 2. Derived from Parasuraman et al. (2000) showing process and guidelines on developing automation technology.

In other words, a system designer should start by focusing on the existing human workflow and information requirements - perhaps broken down into the different information processing types, goals, and roles of the operator(s). Following that, one can propose various automation levels and degrees, to include whether the system should be adaptive, adaptable, or some other mixed-initiative system in order to improve the overall task process. The key there is to have an experienced human factors team to evaluate the consequences of different configuration with measures such as situational awareness, trust, attention allocation, and mental workload all of which are influenced by automation.

Finally, other aspects can be incorporated such as understanding the influence of different reliability levels and the cost of different types of mistakes (humans and automation both create these). One critical aspect of the evaluation should be failure detection. An initial attempt at defining areas or processes for candidate automation could come from widely published, and high-level, 'kill chains' [58] in offense, and in defense.

Additional detail for particular steps in an intrusion kill chain may be drawn from the ample literature on 'attack graphs' which detail individual steps taken for steps in the kill chain. This sets some boundaries for design and for operator assessment. Generating ties to operator information processing (e.g., is the operator taking information in - recon, or making decisions about what to do next) should further shape design of automated cyber operations.

Defensive tactics could be viewed and categorized according to information processing levels used by various blue team members. Then, one can use the Parasuraman et al. (2000) framework to consider different, desirable degrees of automation as related to each defense tactic. While difficult in the abstract, when given the specific network context of "Detection" or "Degradation" as a Blue Team mission, it may be tractable.

\section{Recommendations}

Below are several recommendations, the first primarily concerned with the design and design process surrounding developing automation for cybersecurity. As discussed previously, these are general guidelines.

Automation performance requirements: Make automation performance as high as possible without sacrificing diagnostic transparency. In essence the correct performance is that of the combined human and automation, not one or the other in isolation [6]. The more automation is making decisions or taking action, the more accurate it needs to be in order for it to actually provide improvement to the system as a whole [27], while automating information acquisition and assessment or analysis stages should be at least $70 \%$ accurate [28]. 
Don't hide information, if possible: Do not hide the raw data from users who need it, or at least, test whether this is $\mathrm{OK}$ to do under both normal and under failure conditions. Otherwise the system is encouraging blind trust in automation and complacency.

Ensure that users have clear explanations for how data is gathered, assessed, decided, or acted upon. Similar to the above, this form of diagnostic transparency is common in systems used for purposes of diagnosis by technical experts, but is often stripped out or hidden in order to convey superficial simplicity to other types of users. Ensure the purpose, function, and scope of the automation is communicated clearly and accurately to the user - let them know whether it is operating in or outside of the window of the designer's intentions [59]. Make the source of any potential automation failure clear: slow degradation usually prevents people from recognizing that the situation is changing and that the automation may be malfunctioning [56].

Consider how users will interrupt or change automation performance: Ensure operators can do more than plan. Make sure automation, while being effective under normal conditions, also allows its users to revise and re-plan actions of importance.

The next set of recommendations are research and experimentally focused, derived from our own expertise, and the existent literature available to us at this time, in addition to the gaps identified in this paper. Research need 1: there is a need to generate more, novel and specific evidence that the general approach to automation and its principles applies to cybersecurity. Partially that is our motivation in writing this paper; we hope to have given enough links and examples that future interested parties have a better starting point to do so. This will help refine and improve those recommendations. Research need 2: a more rigorous review of available cybersecurity and automation literature should be conducted to ascertain the state of the art. While we believe there to be little evidence of widespread use, additional practitioner perspective and anecdotal evidence could be incorporated into best design and development practices. There are available methodologies for conducting literature reviews of this sort that could be selected to fit these needs.

\section{Conclusions}

Automation for cybersecurity is possible; but the philosophy of design of the automation should seek to support human decision making. This is easier said than done of course, as we acknowledge above many times this is the hard part of the design decisions.
While some cyber tasks could be reconfigured from the ground up, thus creating a new task for new people to learn and become expert at, many cyber operations are continuous 24/7/365 tasks that have enormous enterprise inertia. They cannot be 'halted' and changed in major ways without at least the perception if not the reality of major risk to mission.

A first step is thinking of humans as your most effective resource, and focusing on deeply understanding what they know, how they work, and what they are good at across time. The more that automation plays a central role, the less of this flexibility will be available, resulting in systems which are both brittle and inscrutable. The obvious is that designing for and with users will mean more use of the system by them, and hopefully more efficient operations as a result. The best method of development and delivery is an iterative and interactive one. Early incorporation of a dedicated professional human factors expert, clear enumeration of the goals of the project and the tasks and goals of the user (as well as the automation) can be leveraged toward developing a useable system in the processes of Figure 2. Combined with the phases of a kill chain, or the defensive goals $d u$ jour, this would create powerful automated products and technology that would ensure avoidance of the 'ironies' and improve the state of security. The iteration in design and development incorporates the actual user base who will go on to use the system; an expert human factors professional is used to determine and weigh the user feedback and interactions on prototype devices and recommend changes to new iterations. Even the measurements may change and evolve over time, especially in the case of situational awareness, which is very contextually based [57]

There are also existing, tested and proven methods for understanding user roles, goals and mental methods for performing work that are approachable for nonhuman factors professionals to understand. As Pritchett explains, the process is not over just by getting user feedback, but requires using methods for testing humanautomation configurations: in other words, don't just hope to get it right, measure it and find out the pros and cons.

Over-automated systems are difficult to navigate and diagnose by humans, suggesting that simple and powerful solutions for problems that are still on a "human" time scale may be the best general design concept available to us today. To develop more nuanced guidelines will probably require, to some extent, intelligently planned trial and error [22]. However, combined with avoiding the ironies, improved design methodology, and heavy incorporation of human factors professionals, we believe great progress can be made in cybersecurity. 


\section{References}

[1] W. Newhouse, S. Keith, B. Scribner, and G. Witte, "National initiative for cybersecurity education (NICE) cybersecurity workforce framework," NIST Spec. Publ.,. 800, 2017.

[2] W. U. Hassan et al., "Nodoze: Combatting threat alert fatigue with automated provenance triage.," Netw. Distrib. Syst. Secur. Symp.

[3] S. Ruoti et al., "'We're on the same Page": A usability study of secure email using pairs of novice users," Proc. CHI . 4298-4308, 2016.

[4] K. M. Ramokapane, A. Rashid, and J. M. Such, “"'I feel stupid I can't delete...": A study of users' cloud deletion practices and coping strategies," SOUPS 2017, no. 241-256, 2017.

[5] R. Parasuraman and V. Riley, "Humans and automation: Use, misuse, disuse, abuse," Hum. Factors, vol. 39, no. 2, pp. 230-253, 1997.

[6] H. R. Booher, Ed., Handbook of human systems integration. John Wiley \& Sons, Inc, 2003.

[7] P. J. Hinds, "The curse of expertise: The effects of expertise and debiasing methods on predictions of novice performance," J. Exp. Psychol. Appl., vol. 5, no. 2, pp. 205-221, 1999 ,

[8] P. Rajivan and N. J. Cooke, "InformationPooling Bias in Collaborative Security Incident Correlation Analysis," Hum. Factors, vol. 60, no. 5, pp. 626-639, 2018,

[9] R. S. Gutzwiller, K. J. Ferguson-Walter, and S. J. Fugate, "Are Cyber Attackers Thinking Fast and Slow? Exploratory Analysis Reveals Evidence of Decision-Making Biases in Red Teamers," Proc. Hum. Factors Ergon. Soc. vol. 63, 2019.

[10] J. K. Hawley, A. L. Mares, and C. A. Giammanco, "The Human Side of Automation: Lessons for Air Defense Command and Control," Army Res. Lab Tech. Rep. 3468, 2005.

[11] US Navy, “(U) Collision report FITZGERALD and MCCAIN," Memorandum, 2017.

[12] T. C. Miller, R. Faturechi, and M. Rose, "Disaster in the Pacific: How We Investigated the Navy's Twin Disasters in the Pacific," Propublica, Feb. 2017.

[13] T. Sheridan and E. Nadler, "A review of human-automation interaction failures and lessons learned," US Dep. Transp., 2006.

[14] B. Strauch, "Ironies of Automation: Still Unresolved After All These Years," IEEE Trans. Human-Machine Syst., 48, 5, p. 419-33, 2017 ,

[15] R. S. Gutzwiller, S. Fugate, B. D. Sawyer, and
P. A. Hancock, "The human factors of cyber network defense," Proc. Hum. Factors Ergon. Soc., vol. 59, no. 1, pp. 322-326, 2015

[16] Y. Acar, S. Fahl, and M. L. Mazurek, "You are Not Your Developer, Either: A Research Agenda for Usable Security and Privacy Research Beyond End Users," 2016 IEEE Cybersecurity Dev. (SecDev), pp. 3-8, 2016.

[17] N. Patnaik, J. Hallett, and A. Rashid, "Usability smells: An analysis of developers' struggle with crypto libraries," SOUPS 2019, 2019.

[18] T. B. Sheridan and R. Parasuraman, "HumanAutomation Interaction," Rev. Hum. Factors Ergon., pp. 89-129, Jan. 2005

[19] S. W. A. Dekker and D. D. Woods, "MABAMABA or Abracadabra? Progress on HumanAutomation Co-ordination," Cogn. Technol. Work, vol. 4, no. 4, pp. 240-244, Nov. 2002.

[20] A. Chapanis et al., Human engineering for an effective air-navigation and traffic-control system. 1951.

[21] T. B. Sheridan and W. L. Verplank, "Human and computer control of undersea teleoperators," ManMachine Syst. Lab, MIT. 1978.

[22] T. B. Sheridan, "Comments on 'Issues in HumanAutomation Interaction Modeling: Presumptive Aspects of Frameworks of Types and Levels of Automation' by David B. Kaber," J. Cogn. Eng. Decis. Mak., 12, 1, p. 25-28, 2017.

[23] M. R. Endsley and D. B. Kaber, "Level of automation effects on performance, situation awareness and workload in a dynamic control task," Ergonomics, 42, no. 3, pp. 462-492, 1999.

[24] R. Parasuraman, T. B. Sheridan, and C. D. Wickens, "A model for types and levels of human interaction with automation.," IEEE Trans. Syst. Man Cybern. Part A Syst. Humans, vol. 30, no. 3, pp. 286-297, May 2000.

[25] T. B. Sheridan and R. Parasuraman, "Humanautomation interaction," in Reviews of Human Factors and Ergonomics, 2005, pp. 89-129.

[26] J. Boyd, "Winning \& Losing," (Unpublished Lect. Notes), 1995.

[27] L. Onnasch, C. D. Wickens, H. Li, and D. Manzey, "Human performance consequences of stages and levels of automation: An integrated meta-analysis," Hum. Fctrs, 56, 3, 476-88, 2014

[28] C. D. Wickens and S. R. Dixon, "The benefits of imperfect diagnostic automation: A synthesis of the literature," Theor. Issues Ergon. Sci., vol. 8, no. 3, pp. 201-212, 2007.

[29] L. Bainbridge, "Ironies of automation," Automatica, vol. 19, no. 6, pp. 775-779, 1983.

[30] R. S. Gutzwiller, B. A. Clegg, and J. G. Blitch, "Part-task training in the context of automation: 
Current and future directions," Am. J. Psychol., vol. 126, no. 4, pp. 417-432, 2013.

[31] E. D. Heggestad, B. A. Clegg, A. Goh, and R. S. Gutzwiller, How Cognitive Ability and Automation Influence Training Performance and Transfer. 2012.

[32] J. Cordle, "Design systems that work for people," Proc. - United States Nav. Inst., vol. 144, no. September, pp. 18-22, 2018.

[33] G. Sadowski, C. Neiva, T. Bussa, and C. Lawson, "Market Guide for Security Orchestration, Automation and Response Solutions.," Gart. Inc., no. June, 2019.

[34] D. Murdoch, "2020 SANS Automation and Integration Survey," May 18, 2020.

[35] N. Moray, "Monitoring behavior and supervisory control," in Handbook of Perception and Human Performance, K. Boff, L. Kaufman, and J. Thomas, Eds. New York: Wiley, 1986, pp. 1-51.

[36] R. Parasuraman and D. H. Manzey, "Complacency and bias in human use of automation: An attentional integration," Hum. Factors, vol. 52, no. 3, pp. 381-410, Oct. 2010,

[37] J. S. Warm, R. Parasuraman, and G. Matthews, "Vigilance requires hard mental work and is stressful," Hum. Factors, 50, 3, p. 433-41, 2008.

[38] M. R. Endsley, "Situation awareness: operationally necessary and scientifically grounded,"Cog. Tech. Work, 17, 2, 163-7, 2015.

[39] M. R. Endsley, "From Here to Autonomy : Lessons Learned From Human - Automation Research" S. A. Technologies

[40] M. R. Endsley and B. Strauch, "Automation and situation awareness: The accident at Cali, Columbia," Proc. 9th ISAP, 1997.

[41] M. R. Endsley and E. O. Kiris, "The out-of-theloop performance problem and level of control in automation," Hum. Fctrs, 37, 2, p. 381-94, 1995.

[42] M. R. Endsley, "Situation Awareness Misconceptions and Misunderstandings," $J$. Cogn. Eng. Decis. Mak., 9, 1, pp. 4-32, 2015,

[43] M. R. Endsley, "Measurement of situation awareness in dynamic systems," Hum. Factors, vol. 37, no. 1, pp. 65-84, 1995.

[44] C. Kruegel and W. Robertson, "Alert verification determining the success of intrusion attempts.," in DIMVA, 2004.

[45] T. Bass, "Intrusion detection systems and multisensor data fusion: Creating Cyberspace Situational Awareness," Commun. ACM, vol. 43, no. 4, pp. 99-105, 2000.
[46] R. Gutzwiller, "Situation Awareness in Defensive Cyberspace Operations: An Annotated Bibliographic Assessment Through 2015," NIWCTR-3184, 2019.

[47] R. S. Gutzwiller, J. Dykstra, and B. Payne, "Gaps and opportunities in situational awareness for cybersecurity," Digit. Thrt. Res. Pract., 2020.

[48] D. Staheli et al., "Visualization evaluation for cyber security: trends and future directions," Proc. Work. Vis. Cyber Secur., 49-56, 2014.

[49] W. Harris, P. Hancock, E. Arthur, and J. Caird, "Performance, workload, and fatigue changes associated with automation," Int. J. Aviat. Psychol., vol. 5, no. 2, pp. 169-185, 1995.

[50] M. of I. and Transports, "Cruise Ship COSTA CONCORDIA, Marine casualty on January 13, 2012 Report on the safety technical investigation.,' p. 181, 2012.

[51] Exabeam, "2020 State of the SOC Report," Exabeam Res. Reports.

[52] D. B. Kaber, "Adaptive Automation," in Oxford Handbook of Cognitive Engineering, J. Lee and A. Kirlik, Eds. Oxford University Press, 2013.

[53] T. de Greef, H. Lafeber, H. van Oostendorp, and J. Lindenberg, "Eye movement as indicators of mental workload to trigger adaptive automation," Int. Conf. Found. Augment. Cog., 219-28, 2009.

[54] Y. Chu and W. Rouse, "Adaptive allocation of decisionmaking responsibility between human and computer in multitask situations," IEEE Trans. Syst. Man Cybern., 9,12, p769-778, 1979.

[55] A. R. Pritchett, S. Y. Kim, and K. M. Feigh, "Measuring Human-Automation Function Allocation," J. Cogn. Eng. Decis. Mak., vol. 8, no. 1, pp. 52-77, Jun. 2013,

[56] N. B. Sarter and B. Schroeder, "Supporting decision making and action selection under time pressure and uncertainty: The case of in-flight icing.," Hum. Factors, 43, pp. 573-583, 2001,

[57] M. R. Endsley and D. Jones, Designing for situation awareness: An approach to humancentered design, 2nd ed. New York: CRC Press, 2012.

Acknowledgements: We thank Dr. Kimberly FergusonWalter for her tremendous support, for organizing the minitrack, and for her expertise and input on our work, and Dr. Sunny Fugate for numerous discussions with us on this topic.

Disclaimer: Any opinions, findings, conclusions, or recommendations expressed in this publication are solely those of the authors and do not necessarily reflect the views of the Department of Defense or any government agency. 